\title{
Development, testing and implementation of the pantograph damage assessment system (PANDAS)
}

\author{
A. Daadbin ${ }^{1} \&$ J. Rosinski ${ }^{2}$ \\ ${ }^{I}$ School of Computing Engineering \& Information Sciences, \\ Northumbria University, UK \\ ${ }^{2}$ Transmission Dynamics, $U K$
}

\begin{abstract}
Pantograph failures due to complex interactions between the overhead line (OHL) and pantograph structure cause significant problems to the railway industry worldwide. Despite many efforts undertaken worldwide, no successfully implemented pantograph monitoring system has, up until now, been introduced for long-term operation on routinely operating trains.

This paper describes the development, design and test results from the first fully proven Pantograph Monitoring System, which is now deployed on routinely operating trains in the UK.

The system uses two subcomponents: the Digital Processing Module (DPM), which is directly clamped on the live $25 \mathrm{kV}$ pantograph structure, and the Receiving Signal and Relay Unit (RSRU) which is installed in a secure location inside the carriage. A pantograph mounted unit is interfaced with the accelerometers that are attached in vicinity of the carbon strip. The DPM uses Bluetooth communication to report any unexpected events to the RSRU. The DPM has an on-board GPS module and acquires and stores time domain data corresponding to the 100 highest events captured during daily train operation. The data is downloaded to the RSRU on a daily basis. Any high alarm events are instantaneously transferred to the train to warn the operator and the control centre about potentially a harmful event that requires immediate attention. The 'hot spots' caused by the overhead line are mapped and trended to allow successful implementation of predictive maintenance of the OHL. The system uses the GPRS mobile network to allow instantaneous access and remote interrogation from any location worldwide.
\end{abstract}


The system described in this paper represents the newest developments in pantograph monitoring and it is now in routine operation.

Keywords: pantograph failure, monitoring system, condition monitoring, Bluetooth communication.

\section{Introduction}

Pantographs are the single contact point between the rolling stock and the catenary. Good contact must be maintained under all running conditions to ensure seamless collection of power. The higher the speed, the more difficult it is to maintain good contact.

In Europe the overhead line infrastructure is designed for a lifespan of 30-50 years plus. This has resulted in the selection of specific materials, such as pure carbon or copper and graphite impregnated carbon, for the critical pantograph contact strips. However, these materials present the drawback of wearing very rapidly, increasing the need for intense regular maintenance.

Traditionally, European railways support a maintenance strategy based on inspecting and replacing pantograph heads rather than focusing on the overhead infrastructure. Problems with the overhead line during contact with the pantograph strip can promote wear and damage to the pantograph carbon element. There are reports of pantograph heads needing replacement after a single journey on high speed trains. Therefore, a monitoring system for an accurate identification of the overhead line geometry faults and their locations is extremely valuable.

\section{Pantograph environment}

Due to the nature of the environment in which the pantograph operates, it is very difficult to put any monitoring in place. Figure 1 show the role of the pantograph in connecting the supply from the overhead line to the train.

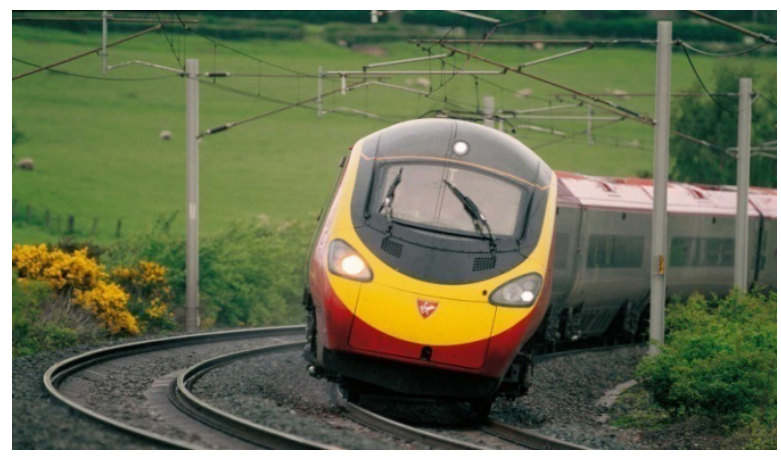

Figure 1. 
In Figure 2 a close-up view of the pantograph is shown at the resting position. When the power needed, the mechanism is raised until the carbon strips get closer to the overhead line and make contact.

Taking measurements of the interaction between the train pantograph and the overhead line catenary has long been a challenge, due to the need to overcome the hostile environment and the problems of isolation. Such measurements require a telemetry system to transmit information from pantograph mounted transducers, at a potential of $25 \mathrm{kV}$, to recording equipment located in the vehicle body.

Transmission Dynamics, in conjunction with Serco Rail, has developed and successfully implemented the Pantograph Monitoring System, which is now deployed on routinely operating trains in the UK. The following section deals with the details of the components involved and typical signals used in on-line monitoring.

\section{Monitoring system}

The system uses two subcomponents. The first subcomponent is the Digital Processing Module (DPM) shown in Figure 3.

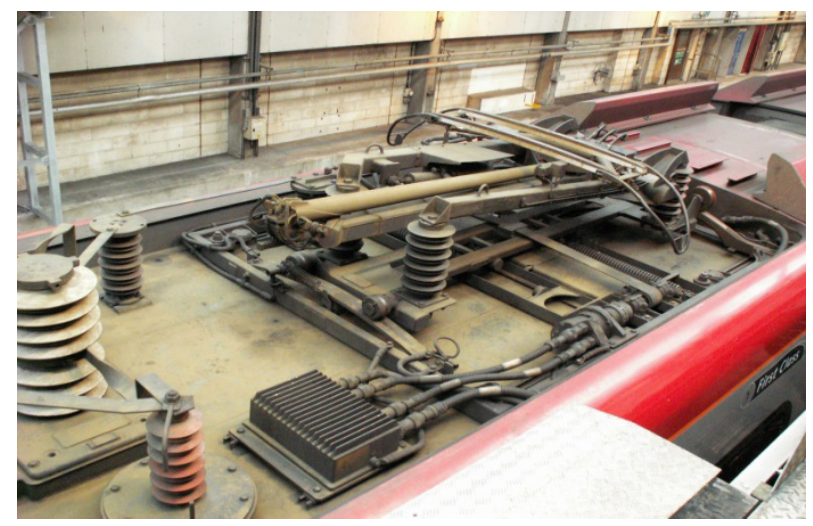

Figure 2 .

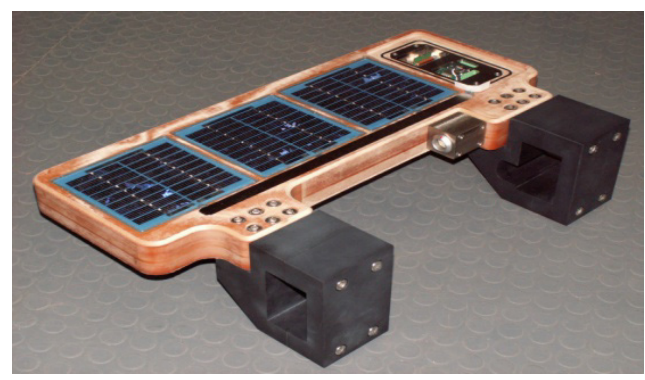

Figure 3. 


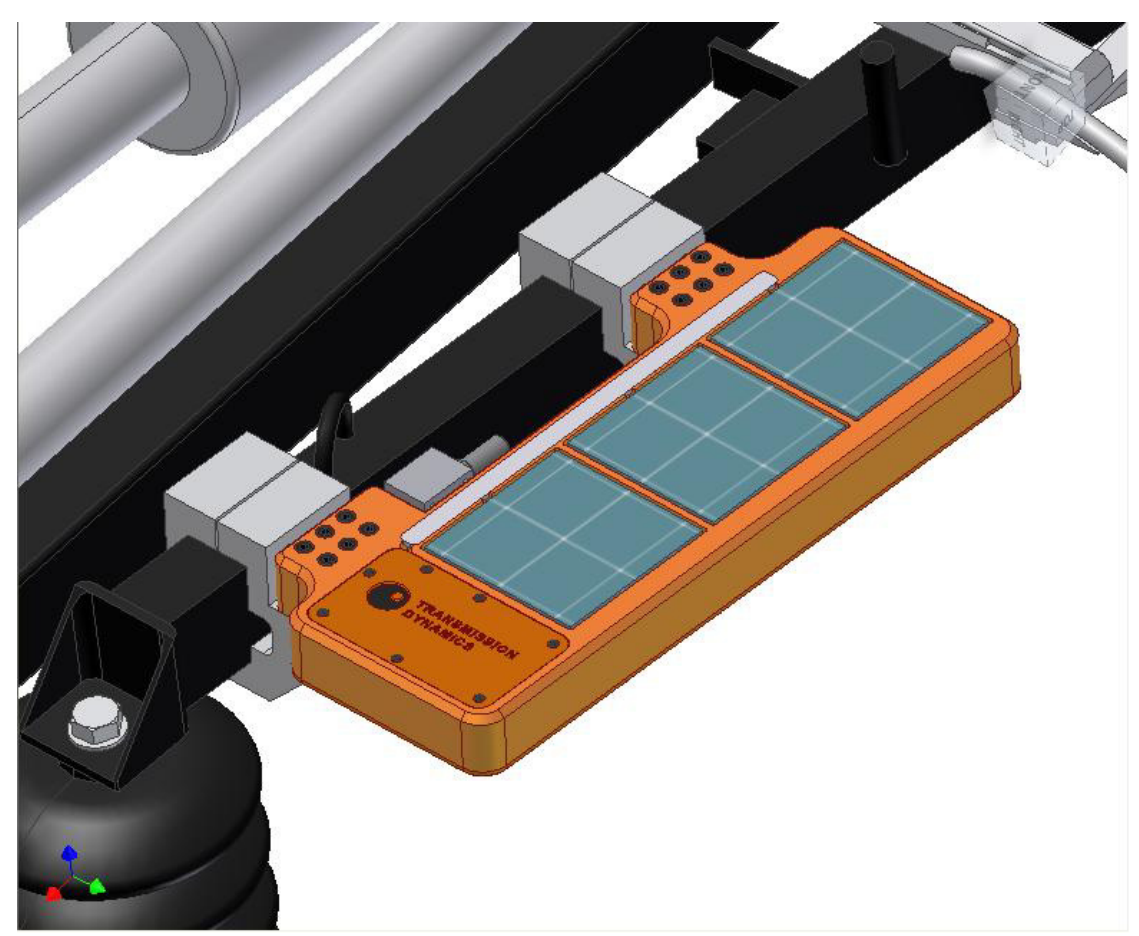

Figure 4.

This DPM is directly clamped on the live $25 \mathrm{kV}$ pantograph structure, as shown in Figure 4. The DPM is interfaced with the accelerometers attached in the vicinity of the carbon strip. The DPM houses integrated battery cells and is equipped with an array of three solar panels. Intelligent power management ensures that batteries are replaced only twice per full year of operation.

The second subcomponent is the Receiving Signal and Relay Unit (RSRU), which is installed in a secure location inside the carriage. The DPM uses Bluetooth communication to report any unexpected events to the RSRU.

The DPM has an on-board GPS module and acquires and stores time domain data corresponding to the 100 highest events captured during daily train operation. The data is downloaded to the RSRU on a daily basis. Any high alarm events are instantaneously transferred to the train to warn the operator and the control centre about potentially harmful events that require immediate attention.

\section{Typical data}

The data collection showed that the signals were free from any electromagnetic interference. The typical signal from the two accelerometers on the carbon strip is shown in Figure 5. It shows the acceleration caused by the impact load on the pantograph. 


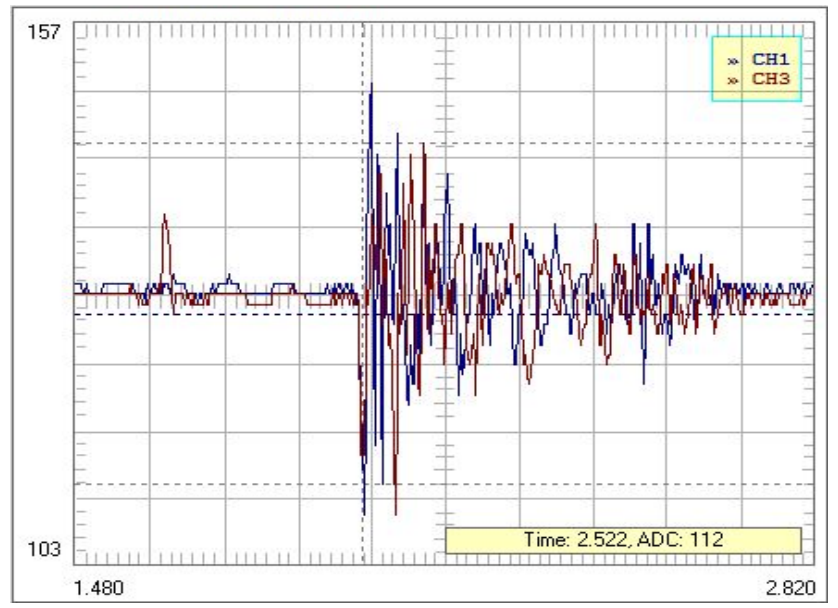

Figure 5.

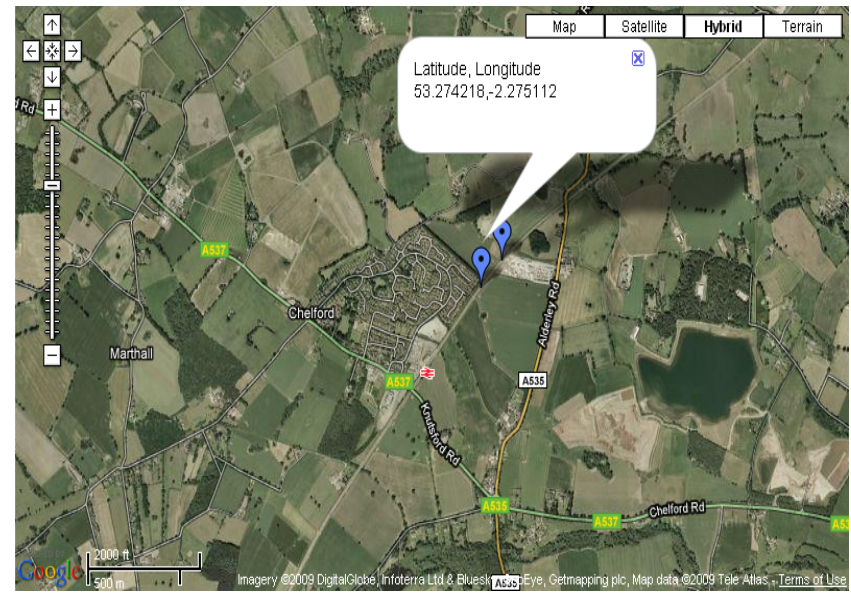

Figure 6: OHL fault location stamp.

The instantaneous monitoring enables the 'hot spots' caused by the overhead line to be mapped and trended to allow successful implementation of predictive maintenance of the overhead line (OHL), Figure 6.

\section{Conclusions}

The Pantograph Damage Assessment System (PANDAS) described in this paper represents the newest developments in pantograph monitoring and it is now in routine operation. Its features are:

- $\quad$ Directly mounted on a $25 \mathrm{kV}$ live pantograph

- Bluetooth radio communication with train 
- On board GPS for accurate event location stamp

- Battery/Soar Panel Powered - long operation life

- On board data storage and signal processing

- Using two accelerometers and two 'dummy' channels

- Overnight download of all events to train mounted SD memory card

- Connected to GPRS mobile network for immediate access and interrogation

- Remotely re-programmable via mobile network

- Robust design - proven in Service

- EMI certified for operation in demanding Rail Environment

This innovative monitoring system reduces the maintenance costs not only for the pantograph but also for the overhead line electrical equipment. 